\title{
Neuroimaging of Visceral Pain
}

\author{
Emily Johns ${ }^{1}$ and Irene Tracey ${ }^{2}$ \\ ${ }^{1}$ Clinical Fellow in Gastroenterology \\ ${ }^{2}$ Professor of Anaesthetic Science \\ Centre for Functional Magnetic Resonance Imaging of the Brain \\ John Radcliffe Hospital \\ Oxford, OX3 9DU \\ Email:emilyj@fmrib.ox.ac.uk
}

\section{SUMMARY POINTS}

- Functional neuroimaging allows conscious reporting by human subjects to be related to changes in brain activation during painful stimulation.

- Brain regions thought to be involved in the perception of pain include the primary and secondary somatosensory cortex, the anterior cingulate cortex, the prefrontal cortex, the insula and the thalamus.

- There are major similarities in how visceral pain and somatic pain are processed by the brain.

- No single brain region has been found to be responsible for visceral pain.

- Patients with IBS often activate the same brain regions as healthy controls in response to pain, but with differing intensities.

- Functional neuroimaging studies have failed to reach a consensus opinion on how the brain processes pain in Irritable Bowel Syndrome.

\section{Introduction}

Modern neuroimaging has revolutionised our understanding of how the brain processes information about pain. Techniques such as functional magnetic resonance imaging (FMRI), positron emission tomography (PET) and magnetoencephalography (MEG) have led to huge advances in our understanding of how pain is perceived by the brain. We know for example, that cognitive factors (such as attention and distraction), context (expectation and beliefs) and mood (depression, anxiety) can all effect how much pain an individual experiences, regardless of the strength of the painful stimulus. However there is also confusion, largely due to the differing techniques used and different populations studied. Pain can be visceral (i.e. from the internal viscera) or somatic (from the soma, or external body), organic or functional, spontaneously occurring or experimentally generated, and studied in healthy volunteers or in patient populations. This review aims to provide a summary of the different neuroimaging techniques available, and to show how these techniques have helped us understand how visceral pain is processed by the central nervous system, with particular reference to the gastrointestinal tract.

\section{Neuroimaging}

Until approximately 15 years ago, our understanding of the role of the brain in pain processing was limited to anatomical and electrophysiological studies in animals. However with the advent of new technologies including PET scanning and FMRI scanning in the late 1970 s, the study of pain in humans became possible. Modern PET scanning relies on the intravenous injection of a radiolabeled isotope, which is then traced in the brain. Differing concentrations and changes in the distribution of isotopes such as $\mathrm{H}_{2} \mathrm{O}^{15}$ and 18-fluorodeoxyglucose, enable the measurement of cerebral blood flow and neuronal metabolism respectively. Since these changes correlate well with regional brain activity, images can be generated that express relative changes in brain activation in response to various stimuli. Similar maps of brain activation can also be created using FMRI, however in this case the maps reflect changes in blood flow which is detected by differences in the magnetic properties of oxygenated and deoxygenated blood.

Although PET and FMRI have good spatial location, they do not have the temporal sensitivity to reflect changes in activation over short time periods such as milliseconds. A newer technique, MEG, is based on recording the magnetic field that is generated by active cortical neurons. This has the advantage of recording neuronal 
activity directly on a millisecond by millisecond basis, with a level of anatomical accuracy comparable to PET and FMRI. In addition to localizing brain activation, MEG may also be able to show the temporal sequence of activation.

\section{Visceral pain differs from somatic pain}

Pain from the internal organs or viscera is often difficult to localise and is rated as more unpleasant than somatic pain ${ }^{1}$. It is described in more emotive language and can result in quiescence and withdrawal as opposed to the active motor 'fight or flight' response usually illicited by somatic pain ${ }^{1,2}$. Classically, these differences had been thought to reflect the differing neuroanatomy of the peripheral nervous system. Information from the skin is transmitted via cutaneous afferent fibres to the dorsal horn of the spinal cord at a specific level according to the dermatome of the nociceptor. From there information is passed via the anterior spinothalamic tract which projects into the lateral and medial thalamic nuclei and onto the cortex. Visceral afferent pathways, on the other hand are transmitted either via the vagus nerve to the nucleus tractus solitarious in the brainstem, or through spinal afferents which follow the path of sympathetic (splanchnic) and parasympathetic (pelvic) neurones to the dorsal root ganglia and synapse in the dorsal horn. Differences between visceral and somatic pain can also occur at the level of the nociceptor, in terms of receptor density, distribution and subtype. Unlike the skin, viscera such as the lung, liver and kidney are completely devoid of pain receptors, so that, for example, tumours are able to grow to large proportions without eliciting the sensation of pain. Visceral afferents are also less specialised than skin nociceptors, and can convey physiological information in addition to pain, such as feelings of nausea, fullness and the urge to defaecate.

\section{Neuroimaging during visceral pain in healthy volunteers}

Given these differences it makes sense that there might be different patterns of brain activation seen during visceral pain compared to somatic pain. In order to test this, researchers have used a variety of stimuli to generate or evoke visceral pain and have described patterns of brain activation in response to balloon distension of the rectum ${ }^{3}$, stomach $^{4}$ and oesophagus ${ }^{5}$, and electrical stimulation of both the rectum $^{6}$ and oesophagus ${ }^{7}$. Following a systematic review of 21 studies including noxious and non-noxious stimulation of both the upper and lower GI tract, Derbyshire ${ }^{8}$ concluded that the prefrontal, primary and secondary somatosensory, mid anterior cingulate, perigenual cingulate, supplementary motor, orbitofrontal and insular cortices were consistently activated during (painful) stimulation of the viscera. In fact, activation patterns during visceral stimulation share many common regions with somatic pain which is generally accepted to include the cingulate cortex, the primary and secondary somatosensory cortices, the insular cortex, the thalamus and the prefrontal cortex 9 . The main differences in the processing of visceral pain when compared with somatic pain, includes its preference for the perigenual anterior cingulate cortex and the absence of the thalamus. The role of the perigenual anterior cingulate cortex in visceral pain is well documented ${ }^{10}$ but the absence of the thalamus is surprising, given its central role in the relay of information from the spinal cord to the cortex. This may well be a false negative result, merely reflecting the fact that activation in the thalamus has not been adequately assessed. Indeed, cerebral blood flow is commonly reported to reduce in the thalamus in PET studies of ongoing pain ${ }^{11}$. Further studies are required to clarify the role of the thalamus in processing visceral pain. Other areas where differences may occur include the primary somatosensory cortex ${ }^{12}$, where less activation is generally reported during visceral pain and which might reflect differences in localizing visceral pain, and the brainstem; one FMRI study found increased activation in the right periaquaductal grey and nucleus cuneiformis bilaterally in response to visceral stimulation, when compared with matched somatic stimulation ${ }^{6}$.

\section{Neuroimaging studies in patients with Irritable Bowel Syndrome}

To date, studies comparing patients with Irritable Bowel Syndrome (IBS) to healthy controls have primarily been descriptive and focused on identifying regional brain activation in response to painful and non-painful stimulation. These experiments rely on the concept of visceral hypersensitivity which was first described in 1973 in patients with IBS to reflect the fact that they demonstrated heightened sensitivity to rectal balloon distension ${ }^{13}$ and up to $60 \%$ of patients with IBS have lower sensory thresholds compared to controls ${ }^{14}$. The first experiment to utilise this difference to compare brain activation during rectal balloon distension between healthy volunteers and patients with IBS, used PET to show reduced activation of the perigenual region of the anterior cingulate cortex and increased activation of the prefrontal cortex in the patient group when compared with healthy controls ${ }^{3}$. However other studies using both PET and FMRI have not been able to report consistent findings. One group in fact, demonstrated the converse with increased, as opposed to reduced, activation in the perigenual region of the nterior cingulate cortex ${ }^{15}$. Discrepancies such as this are not alone in the IBS literature and almost certainly relate to the differing methodologies used (for example PET v FMRI, stimuli using set rectal balloon pressures $\mathrm{v}$ stimuli using set perceptions, and the use of different balloon distension protocols), selection of heterogenous patient groups (failing to discriminate between subtypes of IBS) as well as confounds such as attention, expectation and hypervigilence. Therefore, although neuroimaging studies have been able to demonstrate differences in brain activation between patients with IBS and healthy controls, as well as exploring some of the pathophysiological mechanisms thought to contribute to IBS such as the role of gender ${ }^{16}$ and a history of sexual abuse ${ }^{7}$ on brain activation patterns, to date they have failed to reach a consensus opinion on how the brain processes pain in IBS.

\section{Neuroimaging of other visceral pain states}

Although the vast majority of neuroimaging studies in visceral pain have concentrated on functional disorders such as IBS, it has also been used to study the visceral pain associated with organic 
gastrointestinal disorders such as inflammatory bowel disease and pancreatitis. Neuroimaging has also been used to study the perception of pain from outside the gastrointestinal tract although these studies are currently relatively few at the current time and include brain activation in response to gynaecological pain (vulvodynia) ${ }^{18}$ and urological pain (urge to void) ${ }^{19}$.

\section{New directions}

One application of recent advances in neuroimaging has been to study the effect of treatment interventions. Although there are no analgesics specifically targeted at visceral pain, centrally acting analgesics, antidepressants, and psychological therapies have all been shown to be effective. Recent work has focused on the mechanism of action of these therapies and attempted to identify changes in blood flow to brain regions which may underlie symptom changes, illustrating for example the effect of amitriptyline on brain activation patterns using FMRI, changes in blood flow due to cognitive behavioural therapy and changes in 5-HT3 synthesis as a result of 5-HT3 receptor antagonists (Alosetron) using PET scanning.

\section{Conclusions}

Neuroimaging is a useful research tool that has enabled us to study the interaction between the brain and the gut or other viscera. However the multifactorial nature of pain and functional disorders such as IBS, together with differences in study designs make the interpretation of results difficult. In future neuroimaging studies, it will be important to move from describing differences between healthy volunteers and patients to hypothesis driven studies to explain the pathophysiology of visceral pain.

Understanding how visceral pain is processed, from the level of the nociceptor to the brain, could lead to both the development of novel pharmacological therapies, as well as targeted, and thus more effective treatment of visceral pain conditions.

\section{REFERENCES}

1. Strigo IA, Bushnell MC, Boivin M, Duncan GH. Psychophysical analysis of visceral and cutaneous pain in human subjects. Pain 2002; 97(3): 235-46.

2. Keay KA, Bandler R. Distinct central representations of inescapable and escapable pain: observations and speculation. Exp Physiol 2002; 87(2): 275-9.

3. Silverman DH, Munakata J, Ennes M, Mandelkern M, Hoh C, Mayer E. Regional cerebral activity in normal and pathological perception of visceral pain. Gastroenterology 1997; 112(1): 6472.

4. Ladabaum U. Gastric distention correlates with activation of multiple cortical and subcortical regions. Gastroenterology 2001; 120(2): 369-76.

5. Aziz Q, Andersson JLR, Valind S, Sundin A, Hamdys S, Jones AKP, et al. Identification of human brain loci processing esophageal sensation using positron emission tomography. Gastroenterology 1997; 113(1): 50-9.

6. Dunckley P, Wise RG, Fairhurst M, Hobden P, Aziz Q, Chang L, et al. A comparison of visceral and somatic pain processing in the human brainstem using functional magnetic resonance imaging. J Neurosci 2005; 25(32): 7333-41.

7. Sarkar S, Aziz Q, Woolf CJ, Hobson AR, Thompson DG. Contribution of central sensitisation to the development of noncardiac chest pain. Lancet 2000; 356(9236): 1154-9.

8. Derbyshire, SW. A systematic review of neuroimaging data during visceral stimulation. Am J Gastroenterol 2003; 98(1): 1220 .

9. Apkarian AV, Bushnell MC, Treede RD, Zubieta JK. Human brain mechanisms of pain perception and regulation in health and disease. Eur J Pain 2005; 9(4): 463-84.

10. Vogt BA. Pain and emotion interactions in subregions of the cingulate gyrus. Nat Rev Neurosci 2005; 6(7): 533-44.

11. Derbyshire SW, Jones AK. Cerebral responses to a continual tonic pain stimulus measured using positron emission tomography. Pain 1998; 76(1-2): 127-35.

12. Derbyshire SW. Imaging visceral pain. Curr Pain Headache Rep 2007; 11(3):178-82.

13. Ritchie J. Pain from distension of the pelvic colon by inflating a balloon in the irritable colon syndrome. Gut 1973; 14(2):12532. 
14. Wilder-Smith CH, Schindler D, Lovblad K, Redmond SM, Nirkko A. Brain functional magnetic resonance imaging of rectal pain and activation of endogenous inhibitory mechanisms in irritable bowel syndrome patient subgroups and healthy controls. Gut 2004; 53(11): 1595-601.

15. Mertz H, Morgan V, Tanner G, Pickens D, Price R, Shyr Y, et al. Regional cerebral activation in irritable bowel syndrome and control subjects with painful and nonpainful rectal distention. Gastroenterology 2000; 118(5): 842-8.

16. Berman SM, Naliboff BD, Suyenobu B, Labus JS, Stains J, Bueller JA, et al. Sex differences in regional brain response to aversive pelvic visceral stimuli. Am J Physiol Regul Integr Comp Physiol 2006; 291(2): R268-76.

17. Ringel Y, Drossman DA, Leserman JL, Suyenobu BY, Wilber K, Whitehead WE, et al. Effect of abuse history on pain reports and brain responses to aversive visceral stimulation: an FMRI study. Gastroenterology 2008; 134(2): 396-404.

18. Pukall CF, Strigo I, Binik Y, Amsel R, Khalifé S, Bushnell M . Neural correlates of painful genital touch in women with vulvar vestibulitis syndrome. Pain 2005; 115(1-2): 118-27.

19. Hruz P, Lövblad KO, Nirkko AC, Thoeny H, El-Koussy M, Danuser $\mathrm{H}$. Identification of brain structures involved in micturition with functional magnetic resonance imaging (fMRI). J Neuroradiol 2008; 35(3): 144-9. 